\title{
PRECRACKED PIPE UNDER WATERHAMMER ACTION
}

\author{
S. Brosi ${ }^{1}$, M. Niffenegger ${ }^{1}$, K. Reichlin ${ }^{1}$, E. Kobes ${ }^{2}$ and D. Schrammel ${ }^{3}$ \\ ${ }^{1}$ Paul Scherrer Institute, Villigen, Switzerland \\ ${ }^{2}$ Staatliche Materialprüfungsanstalt, Stuttgart, Germany \\ ${ }^{3}$ Kernforschungszentrum Karlsruhe GmbH, Karlsruhe, Germany
}

\begin{abstract}
We numerically simulate a full scale test in several computational steps with the finite element method and compare all calculated data with the experimental findings. First, we compute the deflection under static loading and the spectrum of eigenfrequencies of an integer piping, attached to a nuclear reactor pressure vessel. Then we consider a sudden pipe break at some distance from the vessel, immediately followed by the undamped closure of a check valve close to the break, and calculate the elastic and plastic transient dynamic response; apart from the break, the piping is still integer. Finally, we consider a circumferential internal surface crack, fairly close to the vessel; after extensive testing of our fracture mechanics calculation procedure we investigate the stress in the crack region under the waterhammer action.
\end{abstract}

\section{INTRODUCTION}

As part of the German HDR Safety Programme, the full scale test series E3I was performed to investigate the load bearing capacity of a highly degraded piping system when subjected to a blowdown-induced waterhammer [1].

The test object (Figure 1) was a $25 \mathrm{~m}$ long pipe with inner diameter of $425 \mathrm{~mm}$, including four elbows and connected to a nuclear reactor pressure vessel (RPV). This system was similar to a boiling water feedwater piping and contained at the cross-sectional plane QP1 a test segment with a semielliptical circumferential internal surface crack of initial arc length $2 \alpha=60^{\circ}$ and initial maximum depths relative to the wall thickness of $a / w=0.3$ and 0.5 in the tests E31.2 and E31.3 respectively.

The tests were carried out under simulated operating conditions: internal pressure $p=9 \mathrm{MPa}$, temperature $T=240^{\circ} \mathrm{C}$. A pipe break, which was simulated by the fracture of two rupture discs, initiated the following chain of processes: pressure drop in the pipe, blow- down, rapid closure of the feedwater check valve, waterhammer and finally heavy pipe oscillations. These oscillations effected in the crack region a high bending load and therefore high axial stresses and strains. In E31.2 resulted a maximum stable crack growth of $1.5 \mathrm{~mm}$ and large plastic deformations in the crack region, whereas in E31.3 a leak developed.

Based on the test data, several institutes are carrying

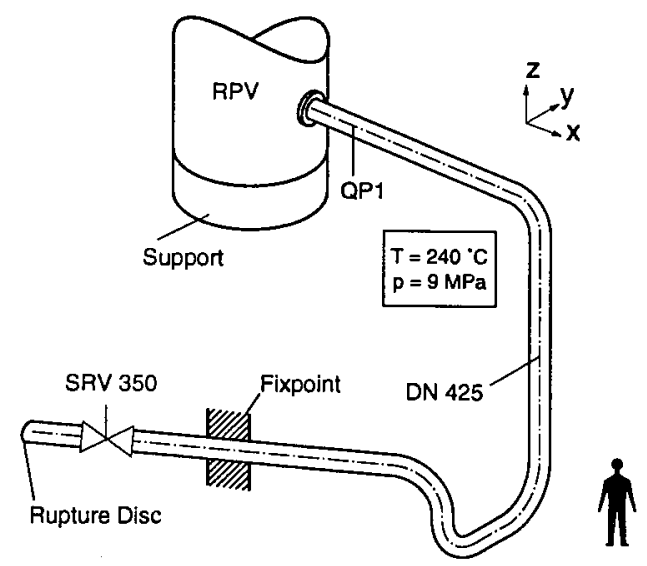

a) Piping System

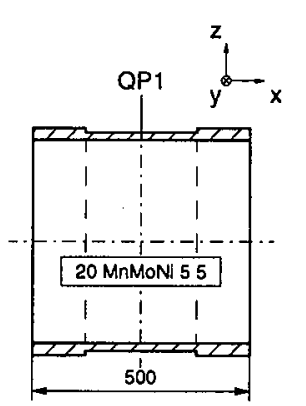

b) Test Piece

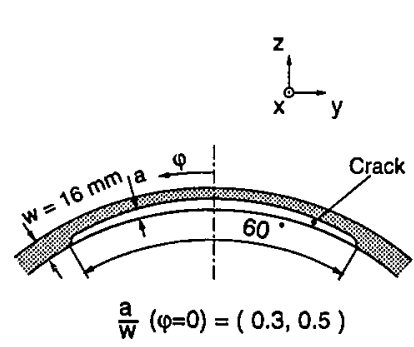

c) Crack Plane QPI
Figure 1: Test facility for the test series E31. 
out an ongoing comprehensive analysis with two objectives, first to numerically simulate the overall reaction of the piping and the local behaviour of the crack zone, and secondly to assess the reliability of different calculation methods by comparison of the calculated results with each other and with the measured values.

At our institutes, with first finite element (FE) calculations [2], which we presented 1991 at SMIRT 11 in Tokyo, we evaluated the global piping behaviour with several simplifications, such as linear elastic material behaviour. Furthermore, we investigated the influence of the variation of the bending axis direction on the stress intensity along the crack front under a uniform crack opening pipe bending load. Already in these first, relatively simple calculations, the qualitative agreement between computed and measured values was satisfactory; however, there emerged unexpected deviations typically e.g. in the pipe deflections.

In the present paper, we report on in-depth research on the global structural behaviour of the piping, which we performed with the aim, among others, to find the causes for these deviations. Furthermore, we analyze the state of stress in the crack region under waterhammer action with fracture mechanics methods, taking account of plasticity.

\section{GLOBAL BEHAVIOUR OF PIPING SYSTEM}

To improve upon the analysis reported in [2] regarding the global behaviour of the piping, we consider two more aspects, the deflection of the piping under static loading and the spectrum of eigenfrequencies; furthermore, in the evaluation of the reactions under waterhammer action we take the plasticity of the material into account. In these additional areas we incorporate also the new results from test E31.3, which was completed in the meantime.

The FE model which we employ here largely corresponds to the one which we used in [2] and described there in detail. The part of the piping system between the RPV and the so-called "fixpoint" is discretized with 59 4-node pipe elements. The pipe end connected to the RPV is rigidly fixed, whereas at the other pipe end the stiff elasticity of the fixpoint is included in the FE model. The influence of the surface flaws on the piping flexibility is considered to be small and therefore is neglected, i.e. the global FE model has no crack.

Presently we use the improved FE program version ADINA 6.0, which (as opposed to the previously used version 5.6) takes into account also the warping of the piping cross-section.

\subsection{Deflection under static loading}

In each case, prior to the actual blowdown tests the piping was subjected to a static vertical force $F_{z}$ (Figure 2) (these tests were called E31.01 and E31.03). For

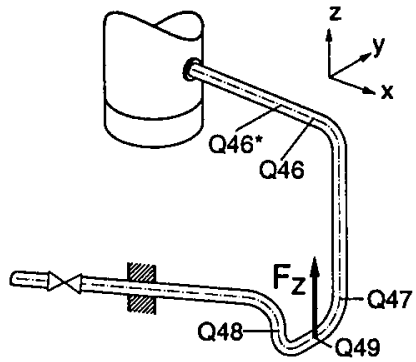

Figure 2: Setup for preliminary tests with static force.

Table 1: Deflection of pipe axis for static loading.

\begin{tabular}{|c|c|c|c|}
\hline \multirow[b]{2}{*}{ Position } & \multicolumn{2}{|c|}{ Displacement $u_{z}[\mathrm{~mm}]$} & Deviation of \\
\hline & $\begin{array}{c}\text { Experiment } \\
\text { E31.01; E31.03 }\end{array}$ & $\begin{array}{l}\text { Calcu- } \\
\text { lation }\end{array}$ & $\begin{array}{c}\text { Calculation } \\
{[\%]}\end{array}$ \\
\hline Q46* & $-; 5.53$ & 4.60 & $\longrightarrow ;-16.8$ \\
\hline Q46 & $9.07 ;-$ & 7.63 & $-15.9 ;-$ \\
\hline Q47 & $10.6 ; 11.0$ & 9.09 & $-14.2 ;-17.4$ \\
\hline Q49 & $10.3 ; 11.1$ & 8.05 & $-21.8 ;-27.5$ \\
\hline Q48 & $6.0 ; 6.22$ & 5.95 & $-0.8 ;-4.3$ \\
\hline
\end{tabular}

Table 2: Eigenfrequencies of piping system.

\begin{tabular}{|c|c|c|c|}
\hline & \multicolumn{2}{|c|}{ Eigenfrequencies [s ${ }^{-1}$ ] } & Deviation of \\
Experiment & Finite Element & Calculation \\
Mode & E31.2; E31.3 & Calculation & {$[\%]$} \\
\hline I & $4.78 ; 4.75$ & 5.11 & $+6.9 ;+7.6$ \\
II & $6.53 ; 6.44$ & 7.05 & $+8.0 ;+9.1$ \\
III & $8.47 ; 8.43$ & 8.93 & $+5.4 ;+5.9$ \\
\hline
\end{tabular}

the load $F_{z}=100 \mathrm{kN}$ Table 1 shows the relative deviation of the computed vertical displacement from the values measured at some representative points on the pipe axis. The positions of the measuring sites Q46, Q46* differ in the two tests for technical reasons; the remaining positions Q47, Q48 and Q49 coincide.

The difference between computed and measured values is minor at the site Q48; however, at all other sites the deviations amount to some $14 \%-28 \%$, and are thus close to the average $18 \%$ which were obtained in [2] for the deviation of the greatest displacement of Q46 under waterhammer action. We therefore conclude that these relatively large deviations in the displacements are in fact due to the greater stiffness of the FE model as compared to the actual structure, and not to measuring errors due to an inadequacy of the displacement sensors for the extreme requirements during the blowdown test.

The static experiments had not been planned from the outset, but it was later decided to perform them, as a confirmation check. When interpreting the results of the static experiments, it must be noted that the displacements are very small as compared to the overall size of the piping, so that their precise measurement is rather difficult, for instance due to manufacturing imperfections. These values are therefore not fully reliable. However, at least qualitatively also this test confirms 
that the FE model is stiffer than the piping.

The mean increase from E31.01 to E31.03 by $5 \%$ of the $z$-displacements at the sites Q47, Q48 and Q49 points towards an additional effect, presumably a softening of the fixpoint beyond its natural elasticity.

\subsection{Eigenfrequencies}

Table 2 shows the first three eigenfrequencies of the piping system, first as obtained in [3] from the displacements and strains measured in the tests E31.2 and E31.3, and secondly as a result from our FE calculation; the last column shows the relative deviation of the computed values. The computed eigenfrequency values generally lie some $7 \%$ above those obtained from the experimental data. Thus again the FE model appears to be stiffer than the model subsumed by the sampled experimental data, as we had already concluded from the smaller deflection in the static tests.

Detailed analysis of the test results [3] shows that the second mode contributes most to the loading on the crack. This shows up here in two respects: in the second mode, the frequency drop from E31.2 to E31.3 (which carries the larger crack) and the deviations of the frequencies directly computed (without crack) from the measured values are largest.

For all modes, the eigenfrequencies of E31.3 are in the mean about $0.5 \%$ lower than those of E31.2; we again ascribe this to an additional softening of the fixpoint, which might have occurred during the test series.

\subsection{Blowdown Test}

Since the overall reactions of the structure were practically identical in the two blowdown tests, and since we do not take into account the cracks in the overall computation, we carry out one computation only, with the loading history obtained from E31.2. The dynamic loading consists of forces which we apply at the elbows and which are calculated from the pipe internal pressure time history recorded in the experiment [2].

In the computation of the influence of the material plasticity on the overall behaviour of the piping under waterhammer action, we employ the true stress-strain curves of the different piping steels, together with von Mises' flow rule and isotropic hardening. Figure 3 shows the properties obtained from specimens of the actual test piece material [4]. Large displacements are taken into account, but due to a program limitation all strains are assumed to be small.

Figure 4 shows the projection of the path followed by the center of the cross-section $\mathrm{Q}^{4} 6^{*}$ onto the $y-z$ plane, i.e. normal to the pipe axis, during the first 150 ms. The centerpoint moves on a clockwise oriented curve first up then down to its maximum deflection. The calculated paths (assuming either linear elastic or elasto-plastic material behaviour) do not differ by much from each other, even the maximum displacements are close. We interpret this to indicate that the difference (reported upon in [2]) between measured and calculated displacements does not differ significantly for the two calculations, thus taking plasticity into account improves the results only by little.

More conspicuous differences between the calcula-

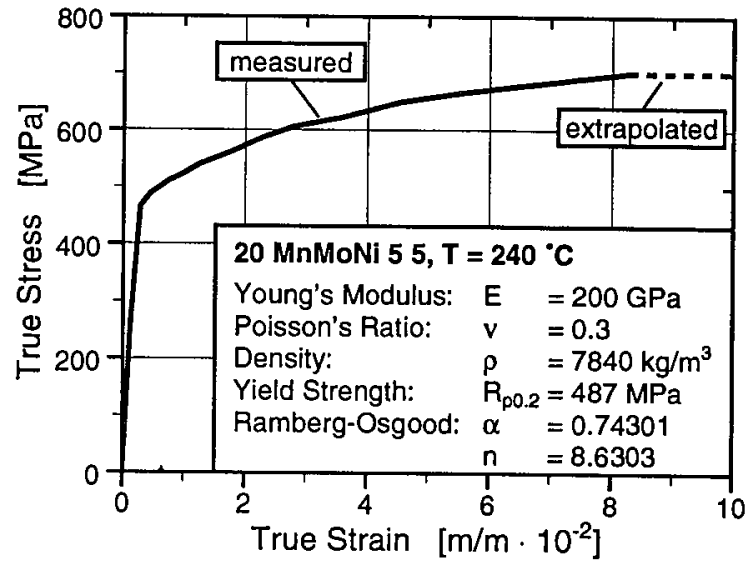

Figure 3: Material properties of test piece.

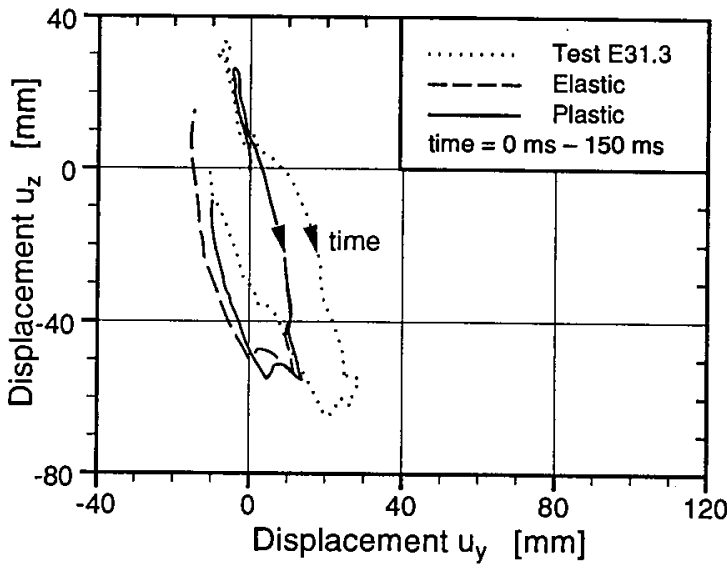

Figure 4: Path of center of Q46* in the $y$-z-plane.

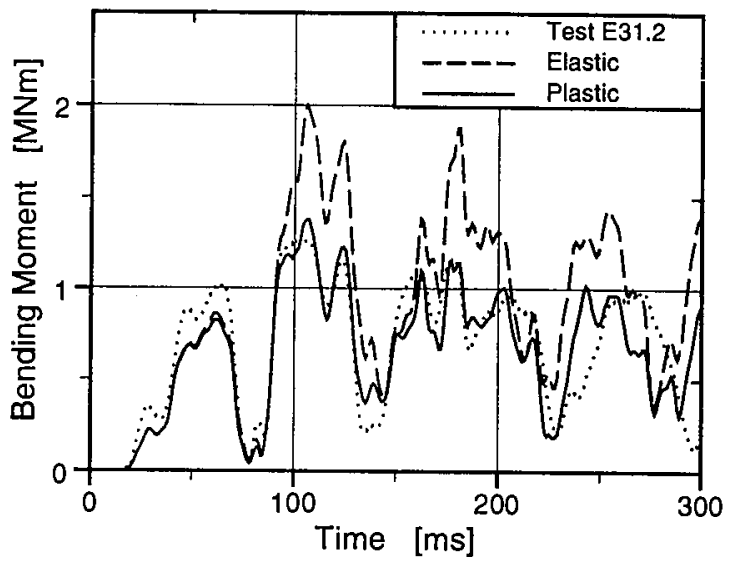

Figure 5: Resultant bending moment in QP1. 
Table 3: Stress intensity factors for uniform loading.

\begin{tabular}{|c|c|c|c|c|c|}
\hline \multirow{2}{*}{$\boldsymbol{w} w$} & $N_{x}$ & $M_{y}$ & \multicolumn{3}{|c|}{$K_{I}[\mathrm{MPa} \sqrt{m}]$} \\
\hline $\mathbf{M N}]$ & {$[\mathrm{MNm}]$} & Murakami & EPRI & FE \\
\hline $\mathbf{0 . 3 0 0 0}$ & 1 & 0 & 7.535 & 7.304 & 7.472 \\
& 0 & 1 & - & 64.8 & 65.21 \\
\hline $\mathbf{0 . 5 3 7 5}$ & 1 & 0 & 12.67 & 11.97 & 12.33 \\
& 0 & 1 & & 107.6 & 108.2 \\
\hline
\end{tabular}

tions with different material behaviour emerge in the resulting bending moment at cross-section QP1 (Figure 5). While increasing towards the first relative maximum, which correlates in time with the first upwards motion of the center of Q46*, the stress at QP1 remains within the linear elastic range, so the calculated moments are still identical. The absolute values of the computed moments are lower than those obtained from the measured strains. The first substantial difference between the two calculations emerges in the time interval $90 \sim 130$ ms. During this phase, which correlates in time with the maximum displacement of Q46*, the maximum loading of the test piece induces a substantial plastification. QP1 is fully plastified and thus not able to carry a higher bending moment anymore. This effect is very accurately computed when taking plasticity into account, since the material parameters are well known; it is of course ignored altogether in the elastic calculation.

Since during the first $70 \mathrm{~ms}$ both the computed displacements at Q46* and the resulting bending moment at QP1 remain below the measured values, it might be the case that the waterhammer loading was not fully taken into account in the calculation. However, it must be allowed that also the bending moment as obtained from the measurements might be in error, since it is interpolated from adjacent cross-sections which are under loading in the linear elastic range. A detailed, direct comparison between computed and measured strains probably would lessen these uncertainties, but so far we were unable to perform it.

\section{CRACK ANALYSIS}

\subsection{Calculation Model}

When we started to compute the state of stress in the crack region, the nonlinear material behaviour as we take it now into account led to excessively high computing times. Therefore, we had to revise the very finely discretized FE model employed in the previous computations [2].

Presently a short, straight pipe segment with an internal circumferential surface crack, inner diameter $d_{i}=$ $425 \mathrm{~mm}$, wall thickness $w=16 \mathrm{~mm}$ and length $l=1000$ $\mathrm{mm}$ is discretized with 42420 -node brick elements and $2314 / 2488$ nodes in the elastic/plastic case (Figure 6).

The crack plane QP1 is one end of the model, since it is now a symmetry plane, so that only one half of

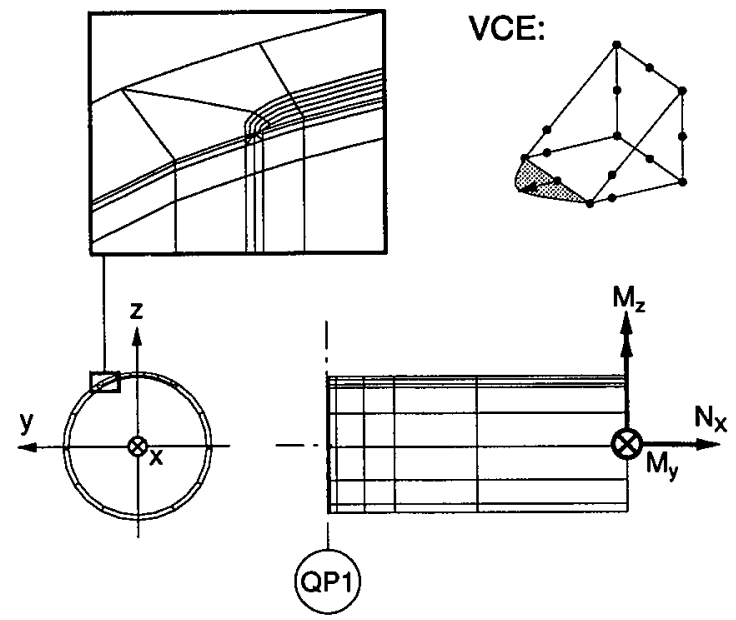

Figure 6: FE model for crack analysis.

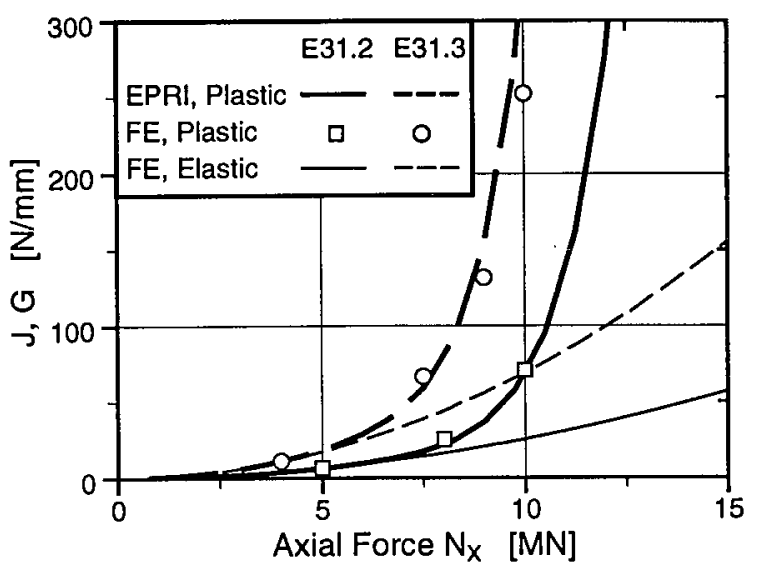

Figure 7: $J$-integral values for axial tension load.

the piece must be modelled. We apply the load at the other pipe end by means of a linearly varying pressure loading to model the cross-sectional stresses, so that we can apply both bending load and tension.

The crack front is modelled by an eccentric circular arc, completed by a quarter of an ellipse at each end. The whole calculation was done for each of the two crack sizes. The largest crack depths correspond to the depths of the initial cracks introduced by fatigue, as they were measured with fractography after the blowdown tests. They turned out to differ somewhat from the planned depths, being $a=4.8 \mathrm{~mm}$ resp. $a / w=0.3$ for the test E31.2 and $a=8.6 \mathrm{~mm}$ resp. $a / w=0.5375$ for the test E31.3.

The crack tip zone is modelled by six circumferential wedge elements with a $1 / \sqrt{r}$ singularity in the elastic case and a $1 / r$ singularity in the plastic case. The energy release rate $G$ and the $J$-integral values respectively are computed by the fracture mechanics post processor ORVIRT based on an ADINA 5.6 stress analysis and by use of the virtual crack extension scheme shown in 
Figure 6. The stress intensity factor $K_{I}$ is computed from the energy release rate $G$ by the relationship valid in the plain strain condition.

\subsection{Verification Problems}

Before we attack the waterhammer problem we check the reliability of the programs and of our FE model in simple loading conditions against available handbook solutions.

Table 3 shows the stress intensity factors $K_{I}$ which we computed, assuming elastic material behaviour, under unit force $N_{x}=1 \mathrm{MN}$ and unit moment $M_{y}=1$ MNm. The handbook result given by Murakami [5] is valid for a semielliptical crack with the same maximum depth; it was obtained using «line-spring» elements. The solution by EPRI [6] is valid for a crack with constant depth, which we again matched to the largest value. The agreement between the different solutions is excellent, the FE solution deviates by at most $3 \%$ from the handbook data.

Figure 7 shows the results of the verification test for plastic material behaviour. We computed the $J$-integral values under a monotonically increasing tensile force, and in addition the energy release rate $G$ for elastic material behaviour. The EPRI handbook solution [6] is based on a Ramberg-Osgood stress-strain relation and again assumes a crack with constant depth. The agreement between our FE result and the handbook data is excellent; the computation thus yields reliable results even at high plastification.

\subsection{Blowdown Test}

Since in the blowdown test the crack cross-section essentially experienced bending load, we assume pure bending for the calculation. We chose five characteristic loading levels from the experimentally determined time history of the bending moment at QP1; these we apply in quasistatic increments in the calculation. The fifth level corresponds to the maximum measured bending load. Thus there is no shock; transients and inertia forces are not introduced at all. This is a major assumption of our procedure, and its confirmation is an essential task of our investigations; however, at the moment the question is not yet settled.

Figure 8 shows the $J$-integral values as computed for the test E31.3 against the crack angle $\varphi$ as defined in Figure 1c. The maximum $J$ values are not reached in the midst of the crack $\varphi=0^{\circ}$; thus here again we observe the crack loading asymmetry, which we already studied in depth in [2]. The same figure also shows the interval for the crack initiation value $J_{i}$ of the test piece material, which was determined by using three CT-25 specimens [4]. The maximum $J$ value, $J_{\max }=256$ $\mathrm{N} / \mathrm{mm}$, calculated for the highest load level, is above $J_{i}$ for the test E31.3; on the other hand, for the test E31.2 there results the significantly lower value $J_{\max }$

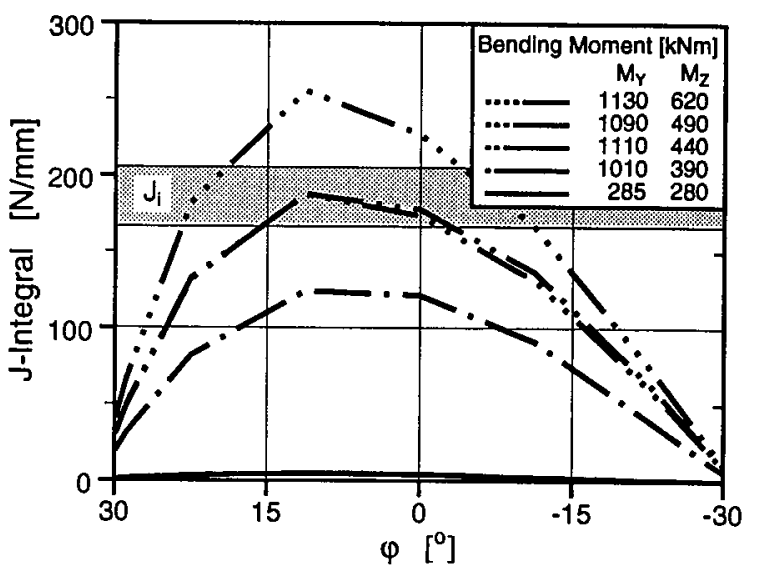

Figure 8: $J$-integral values for blowdown test E31.3.

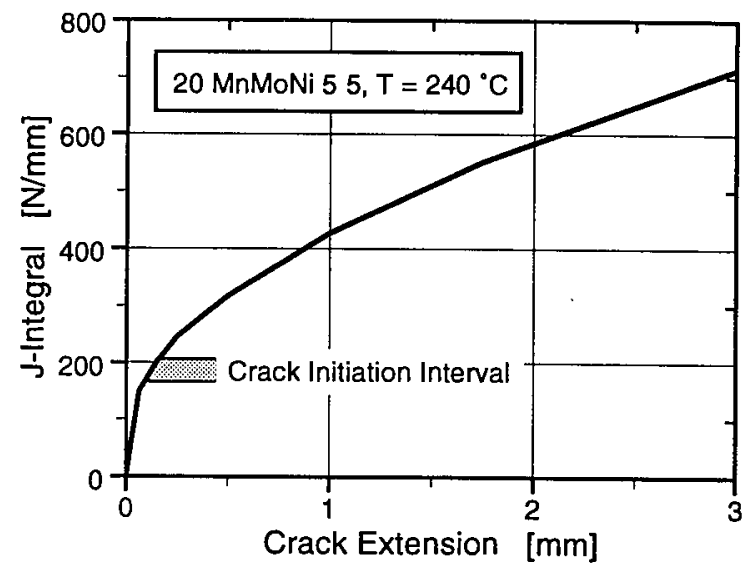

Figure 9: Crack resistance curve for test piece.

$=92 \mathrm{~N} / \mathrm{mm}$, which is even lower than $J_{i}$. In both tests, the cracks grew; thus the comparison of the calculated $J$ values with $J_{i}$ allows a correct prediction of crack growth only in the test E31.3.

If we in addition take into account also the crack resistance curve (Figure 9) for the test piece material [4], we notice that the value $J_{\max }=256 \mathrm{~N} / \mathrm{mm}$ obtained for the test E31.3, under the assumed quasistatic loading, gives rise to a stable crack growth of less than $0.5 \mathrm{~mm}$. This insignificant crack growth is incompatible with the leakage which developed during the experiment. It thus appears that the calculated $J$ values are too low for both tests E31.2 and E31.3.

However, we recall that we are uncertain about the accuracy of the actual bending moment under blowdown conditions. On the other hand, the steep slope of the $J$ curve in Figure 7 shows that at the level of loading which obtains during the waterhammer the $J$ values depend strongly on the absolute loading level. This means that, in addition to the uncertainty in the bending load, also the loads due to the internal pressure and axial tension (which were so far considered to be small and therefore neglected) could have a significant 
effect on the calculated $J$ values.

Thus we conclude that prior to any assessment of the reliability of the quasistatic computation of $J$ as we performed it here, and even more so before any quantitative predictions on crack growth are attempted, we should make sure that the load levels are the same in calculation and experiment, e.g. by direct comparison of calculated and measured strains.

\section{CONCLUSIONS}

We studied the deflection under static loading and the spectrum of eigenfrequencies of an unflawed piping. Then we determined the elastic and plastic global structural dynamic response after a simulated pipe break and undamped closure of a check valve. Furthermore, with a local pipe model which contained a circumferential internal surface crack, we first extensively tested our calculation procedure, and then investigated the stress in the cracked area due to the waterhammer action.

- The global FE model behaved stiffer than the actual system in all respects, in the displacements under static loads, the eigenfrequencies and the waterhammer effect.

- We succeeded in approximating fairly well the characteristic time history of the bending load and of the piping deflection under the waterhammer, by assuming linear elastic material behaviour.

- By taking into account the plastification of the material the differences between computation and measurement were reduced, in the case of the bending moment significantly. So far, we were unable to understand the remaining differences between computation and measurements. Some possible explanations would be, for instance, a different level of loading, the flexibility of the RPV, or some inherent shortcoming of the pipe elements.

- On the other hand, in the field of fracture mechanics we were able to establish the excellent performance of our computation procedure, even under conditions of highly developed plasticity of the material, by comparison with handbook solutions.

- However, the $J$-integral values computed under waterhammer loading as in a quasistatic process are too low; the crack advance computed from them is less than the observed one. Prior to deciding whether the quasistatic approach is adequate, and indeed before using the computed $J$ values to conservatively predict crack growth, we must establish whether the simulation of the waterhammer adopted in the calculation adequately models the actual phaenomenon.

\section{ACKNOWLEDGEMENTS}

We wish to express our gratitude to our colleagues Dr. R. Rösel and G. Duijvestijn from the Paul Scherrer Institute for their support to the present work. We gratefully mention the financial assistance from the Swiss Federal Nuclear Safety Inspectorate and from the Swiss Federal Office of Energy.

\section{REFERENCES}

[1] Kobes E., Kussmaul K., Diem H. and Schrammel D. (1993): «Leak-before-break Behaviour of a Piping System DN425 Subjected to Transient Loading by Water Hammer». Transactions of the 12 th SMIRT Conference, Stuttgart.

[2] Brosi S., Wanner R., Reichlin K., Schrammel D. and Kobes E. (1991): «Structural and Fracture Mechanics Study of a Pipe with a Circumferential Crack under Blowdown-induced Loading». Transactions of the 11 th SMIRT Conference, Tokyo, Vol. F, pp 219-224.

[3] Schrammel D. (1991): «Identifikation der modalen Schwingungsparameter aus den beiden BlowdownVersuchen E31.2 und E31.3». PHDR-Arbeitsbericht Nr. 30.044/91.

[4] Eisele U. and Restemeyer D. (1991): «Werkstoffuntersuchungen zu den Rohrversuchen E21 und E31». MPA-Prüfungsbericht Nr. 875021040 , Staatl. Materialprüfungsanstalt MPA Stuttgart, Universität Stuttgart.

[5] Murakami et al. (1987): «Stress Intensity Factors Handbook». Committee on Fracture Mechanics, The Society of Material Science, Japan, Vol. 2, pp 759-770.

[6] Zahoor A. (1990): «Ductile Fracture Handbook». Electric Power Research Institute, NP-6301-D, Palo Alto, Sections 2.1.1, 2.1.2 and 2.2.1 . 